\title{
Features of Voice Transmission in the LTE Network
}

\author{
https://doi.org/10.31713/MCIT.2019.52
}

\author{
Yevheniy Malanchuk, Andriy Khrystyuk \\ National University of Water and Environmental Engineering \\ Institute of Automatics, Cybernetics and Computer Engineering \\ Rivne, Ukraine \\ a.o.khrystyuk@nuwm.edu.ua
}

\begin{abstract}
The main results of the research of methods of voice transmission in the fourth generation networks, the analysis of existing problems in voice transmission technologies, and also verification of quality and reliability of VoLTE technology in the laboratory environment are presented. Recommendations on the application of voice technology in LTE networks deployed in Ukraine, assessment of existing technologies used for transmitting voice over the fourth generation networks, and the creation of a test strategy for checking the reliability of voice transmission on the LTE network are presented.
\end{abstract}

Keywords - wireless network, data transfer, voice transfer, fourth generation network, LTE

\section{INTRODUCTION}

In the days of modern information technology, the problem of quality voice communication is particularly acute. Fourthgeneration communications technologies are gaining ground, especially surprisingly after the first $5 \mathrm{G}$ non-profit networks. Ukrainian mobile operators are trying to keep up with global trends. Therefore, starting in the spring of 2018, the transition to fourth generation LTE-Advanced networks, the next generation of networks, allowing to achieve high speed data transmission, ensure the stability of the network operation and improve coverage.

Upgrading mobile services has undeniable benefits, however, it has created a number of problems, the main one being voice implementation. As the transmission of information on the fourth generation networks is done through packet transmissions, mobile operators have been faced with the need to introduce new methods for voice services and ensure the quality of voice transmission. This problem is quite acute today, so the presented research is relevant today.

\section{THE PURPOSE OF THE ARTICLE}

To analyse the existing problems in voice transmission technologies, and also verification of quality and reliability of VoLTE technology in the laboratory environment.

\section{MAIN MATERIAL}

3GPP Long Term Evolution (LTE) - next generation $3 \mathrm{G}$ wireless networking technology with high data rate. The relevant standard was developed and approved by 3GPP to provide the required bandwidth and quality of service (QoS) [1].

LTE includes an advanced packet system consisting of an updated UMTS terrestrial radio access network (E-UTRAN) and an improved packet switching center, as well as providing information with high bandwidth, low latency and greater bandwidth through a simplified IP architecture. LTE technology has been developed to work seamlessly with all existing networks to help mobile providers transform their networks to meet user needs. It provides a more efficient and quality service, both for voice and data transmission. LTE has a number of features and advantages over previous cellular standards [2].

The LTE network architecture is designed to support packet traffic with so-called "smooth" mobility, minimal packet delivery delays and high service quality. Mobility as a function of the network is provided by its two types: discrete mobility (roaming) and continuous mobility (handover). As LTE networks must support roaming and handover procedures with all existing networks, LTE subscribers (terminals) must be covered by wireless broadband services.

The network structure is very different from the $2 \mathrm{G}$ and $3 \mathrm{G}$ networks. Figure 1 shows that not only the base station subsystem but also the switching subsystem have undergone significant changes. Data transmission technology between user equipment and the base station has been significantly modified. The data transmission protocols between the network elements have also undergone significant changes. Now all the information, including voice and data, is transmitted as packets. Thus, the division into parts for processing or only voice information or only batch data no longer exists.

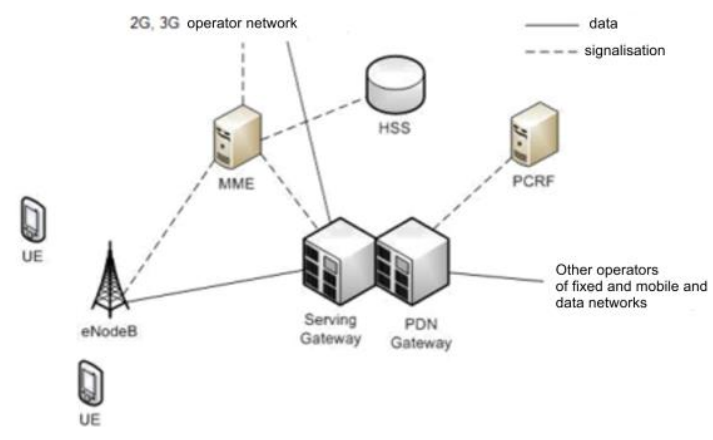

Figure 1. LTE network structure 
Different "channels" are used to transport the data via the LTE radio interface. They are used to highlight different types of data and allow them to be transported through the access network more efficiently. Multiple channels provide a higher level interface within LTE and include clearer and more specific data segregation.

The development of wireless is accompanied by a continuous change in technologies based on GSM and CDMA cellular standards, as well as the standards of IEEE 802 data transmission systems.

Due to the fact that the amount of packet data in the third generation $(3 \mathrm{G})$ cellular networks already exceeds the volume of voice traffic, the very concept of next, fourth generation (4G) networks is inextricably linked (if not synonymous) with creation of universal mobile multimedia information networks. Today, two technology groups are clearly focused on providing universal telecommunications services. This is WiMAX (as a development of the IEEE 802 line) and 4G cellular technology. And each of them occupies a niche in the large wireless market. LTE technology can be deployed on existing 2 and 3 generation networks, which significantly reduces costs than building a network from scratch. Fig. 2 [5] shows the LTE sharing solution and the existing UMTS network.

The development of LTE as a standard officially began in late 2004. The primary purpose of the initial research was to select a physical layer technology that could provide a high data rate. Two options were suggested as the main ones: development of the existing W-CDMA radio interface (used in HSPA) and creation of a new one based on OFDM technology. As a result of the research, OFDM was the only worthy technology, and in May 2006 3GPP created the first specification of the Evolved UMTS Terrestrial Radio Access (E-UTRA) radio interface.

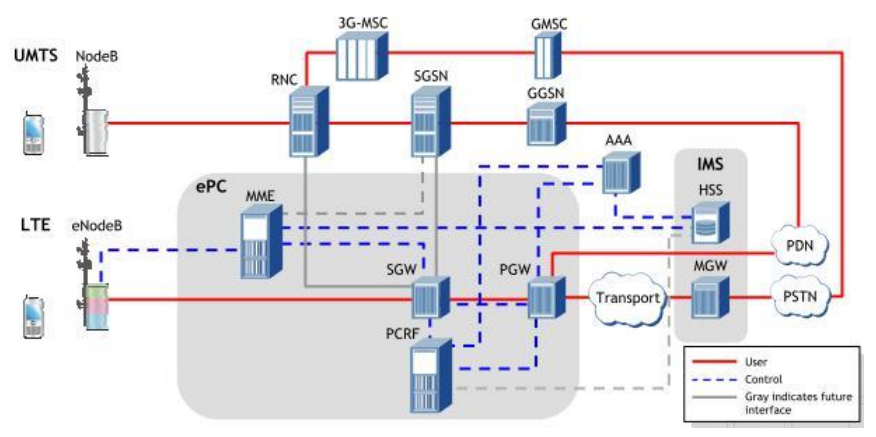

Figure 2. Joint solution of UMTS and LTE cellular technologies

On January 31, 2018, the operators of Kyivstar, Vodafone Ukraine and lifecell received licenses for a frequency of 2600 $\mathrm{MHz}$, which gives a small coverage radius. This allowed the launch of only $4 \mathrm{G}$ spots in April of same year.

The first 4G zones appeared in Lviv, Kyiv, Dnipro, Kharkiv, Odesa, Uzhgorod, Vinnitsa, Khmelnytskyi, Ternopil, but the new connection started working on July 1 (when the $1800 \mathrm{MHz}$ frequency started working). Kyivstar has announced the launch of other non-city points. Among these are popular destinations (Zatoka and others), as well as border crossings.

On March 6, 2018, mobile carriers purchased 4G frequencies in the $1800 \mathrm{MHz}$ band. Total amount received during the auction for $4 \mathrm{G}$ funds in the range of $1800 \mathrm{MHz}$ UAH 5,434 billion. In the course of bidding for frequencies in the range of $1800 \mathrm{MHz}$, the company paid 1.45 billion more than the starting price. The $1800 \mathrm{MHz}$ frequency has only been operational since July 2018, and only then did large-scale $4 \mathrm{G}$ coverage appear in Ukraine. However, in the villages and district centers this frequency is not expected [6].

The further roll-out of VoLTE services may hinder the provision of voice OTT services. To date, there is and is a way of voice communication using OTT (Over-the-Top) services such as WhatsApp, Viber, Telegram, etc. These services are free of charge. The IP-based data network provides convenient transportation for completely different services and brings them out of the operator's area of responsibility to the users themselves. With the advancement of technology (4G), OTT providers are making their services more attractive, for example, providing voice and video resolution for HD.

But this method does not satisfy the users, as these services do not guarantee stable, quality work. The fact is that they rely entirely on the Internet, and online delivery of packages is carried out on the principle of "Best effort" (service of providing connection without guarantees) [7]. Despite the popularity of both trademarks and OTT proprietorship companies, consumers are often unable to take action in the event of poor, unstable operation or even temporary absence. OTT service providers cannot control, more precisely, affect the availability and quality of services. Difficulties also arise with customer support: you need to communicate in a foreign language, and response time depends on the provider's priorities and capabilities.

Every day there are more and more ways to solve the problem of voice over LTE. Analyzing the market situation, more than a dozen such solutions were found. Through analysis, the most promising ways were highlighted, based on world experience:

- $\quad$ Circuit Switched Fall-Back (CSFB);

- $\quad$ Voice over LTE Generic Access (VoLGA);

- $\quad$ IMS-based Voice Services (VoIMS).

Each of these solutions has its advantages and disadvantages.

Differences in the implementation of voice call support on $4 \mathrm{G}$ networks are one of the factors that delay their widespread distribution around the world. Because vendors are interested in promoting "their", developed by the company, or taken as a model of implementation, the completeness of ideas in the field of what are the options, not all experts have cellular operators.

VoLGA and CSFB are an attempt to provide voice services by returning to the domain that is not needed in the all-IP network of the CS domain. Why it is planned - it is clear. These are the re-use of existing equipment, and the 
mechanisms of interconnect and roaming that have been understood and worked out over the years, the possibility of painless integration of the various services that are and are currently working, and the simplicity and transparency of providing inter-system handover from LTE to UMTS / GSM, etc..

Voice over mobile networks are not losing popularity despite the growing popularity of OTT services. In fourthgeneration networks, data transmission is solely based on packet usage, making the implementation of voice over LTE a problematic issue.

The test setup consisted of the MD8475 Anritsu signal tester. The diagram of the test setup is shown in Figure 3. The tester is connected via an RF cable to the shielded RF enclosure. The shielded RF enclosure contains an RF antenna that creates two-way communication with the UE. Once set up, the shielded enclosure is closed to prevent any external interference. The MD8475 switches on or off, changing any settings, such as power level, if required. When simulation is enabled, the radio starts broadcasting inside the shielded enclosure, allowing the UE to join the simulated network.

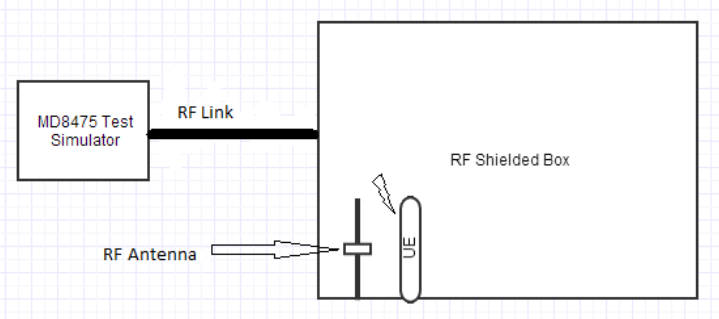

Figure 3. Scheme of the test installation

IMS Droid is an open source application that simulates the IMS / SIP stack along with other required ISIM settings. Installing this application allows you to use a regular LTE device as a VOLTE device. The SIP stack simulated by this application enables the device on which it is enabled to register directly on the S-CSCF server in the IMS tester kernel. Once registered, the program can receive calls from the tester and make calls to the tester using the SIP ID instead of the phone number itself. SIP signaling part and protocol signaling during initial registration remain unchanged. In addition, the connection established for a voice call using this application is not another VoLTE carrier but is established on the same LTE carrier. In addition, the quality of service achieved is often not guaranteed. But despite the difference from a real VoLTE call, there is no effect on the process of pre-registering VoLTE LTE or SIP and signaling during a voice call. This allows us to simulate the VoLTE call on the MD8475 simulator, since the main purpose is to view alarms rather than measure performance.

IMS Droid APP is used to simulate ISIM parameters and to allow non-VoLTE UEs to communicate with the IMS server and create a VoLTE call based on SIP. The scenario under consideration is a VoLTE Mobile Terminated voice call. Initial IMS registration contains the first two messages observed in wireshark, which is a REGISTER request on anritsu-cscf.com, which is a debugged S-CSCF in the
MD8475 simulator. Once the hash request has been agreed with the UE, the UE must be properly authorized in the IMS kernel.

When attempting to end a call from the simulator, we observe the installation of a VoLTE voice call based on SIP. SIP is an application layer protocol that can run on TCP / UDP as the primary transport layer protocol. SIP is designed as a text protocol that is independent of the underlying transport layer structure and can use lower layer protocols. The log segment shown in Figure 4 is intended to call VoLTE from a mobile terminal. SIP alarm in case of UE-IMS interaction during MT VoLTE call sending an INVITE message from the IMS server to the UE. Session identification and reconciliation is performed using SDP. Parameters are described as a session profile or as management parameters that define and establish a communication session. All bandwidth parameters, QoS, service type (sound in this case), port number to be used for communication, and all other defined parameters described in the SDP message and these values are agreed / configured with this message. The UE then sends 100 Trying message and Ringing message messages before actually making the VoLTE call. The UE responds to Provisional Acknowledgement (PRACK) prior confirmations after the call is connected. This acknowledgment is passed to the end UE that initiated the call. In this case, the UE is modeled inside the MD8475 simulator.

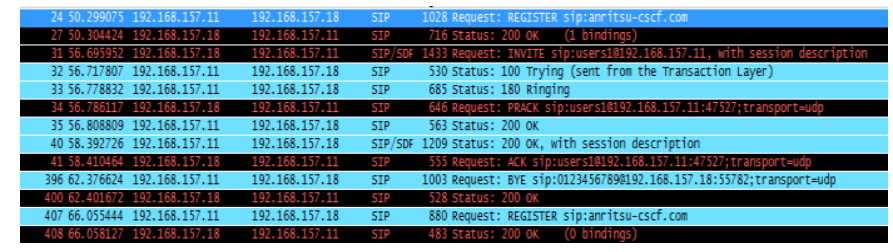

Figure 4. SIP alarm during VoLTE call on MD8475 simulator

Back to back multiple VoLTE calls were made to monitor the average setup time, which provides input on how quickly scripts need to be developed to test the stability of the VoLTE call setup. The Multiple VoLTE Calls alarm is shown in Figure 5. Also, the average installation time for different signals is shown in Table 1.

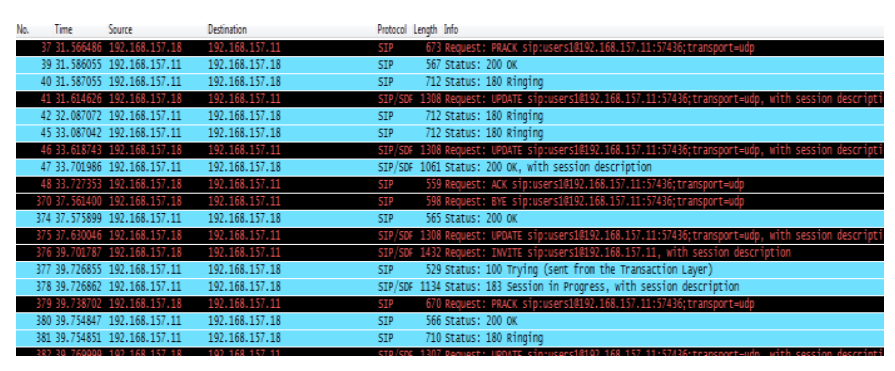

Figure 5. SIP Alarm from Wireshark Test Logs for Multiple VoLTE Calls

It was noted that the average call time for the 10 calls collected in Table 2 is about 0.079 seconds. This gives an idea of how to develop a test script when trying to create concurrent test scenarios. 
TABLE I. VOLTE CALL SETUP TIME IN SECONDS

\begin{tabular}{|l|c|c|}
\hline S.No & $\begin{array}{c}\text { Call Setup Time } \\
\text { (seconds) }\end{array}$ & $\begin{array}{c}\text { DL/UL Reference } \\
\text { Power }(\mathrm{dBm})\end{array}$ \\
\hline 1 & 0.08 & $-40 /-20$ \\
\hline 2 & 0.06 & $-40 /-20$ \\
\hline 3 & 0.08 & $-40 /-20$ \\
\hline 4 & 0.07 & $-40 /-20$ \\
\hline 5 & 0.1 & $-40 /-20$ \\
\hline 6 & 0.07 & $-40 /-20$ \\
\hline 7 & 0.08 & $-40 /-20$ \\
\hline 8 & 0.09 & $-40 /-20$ \\
\hline 9 & 0.08 & $-40 /-20$ \\
\hline 10 & 0.08 & $-40 /-20$ \\
\hline
\end{tabular}

According to research, and after studying the technological aspects of VoLTE, the following test scenarios are identified that are used to measure the stability and performance of the VoLTE service.

1) IMS registration: validation of a device that successfully registers on the IMS server by connecting to the S-CSCF SIP server. A valid APN will be verified and the desired result is successful registration.

2) Extra Services: Extra services such as call forwarding, conference calls, must be verified for VoLTE calling. All other USSD-related tests can be performed to test the performance and stability of VoLTE calls.

3) Mobility scenarios: Mobility scenarios between EUTRAN and GERAN / UTRAN networks during a fixed call to confirm the quality and stability of the calls. Also mobility between LTE coverage areas with and without IMS support.

4) Emergency calls: emergency calls setup, installation time, call quality, stability tested in mobility scenarios, transfer scenarios. Automatic redial mechanisms, back-to-back stability and long-term calls for verification.

5) SMS: It is necessary to explore SMS-related scripts, in the absence of CS domain. SMS transmission and reception must be verified. You should also check different SMS classes and different encoding schemes.

\section{CONCLUSIONS}

The analysis of features of implementation of voice transmission in the LTE network allowed to obtain the following results. LTE wireless technology differs from second- and third-generation high-speed data transmission through the use of Multiple Input Multiple Output (MIMO) technology, OFDM modulation scheme and channel bandwidth increase of up to $20 \mathrm{MHz}$. In fourth-generation networks, data transmission is solely based on packet usage, making the implementation of voice over LTE a problematic issue. LTE Call Modeling Investigates Average Call Set-up Time for a VoLTE Service Using the IMS Platform. Measurement results show that the average time to make a voice call using VoLTE technology over IMS is 0.079 seconds, which is ten times less than the time of a call using Circuit Switched Fall-Back technology currently used in Ukraine.

\section{REFERENCES}

[1] Informatsiyni merezhi chetvertoho pokolinnya 4G. [Elektronnyy resurs]. - Rezhym dostupu: http://stud.com.ua/20632/informatika/informatsiyni_mere zhi_chetvertogo_pokolinnya

[2] Gel'gor A.L., Popov Ye.A., Tekhnologiya LTE mobil'noy peredachi dannykh: Uchebnoye posobiye. - SPb.: Izd-vo Politekhn. un-ta, 2011.- 204 s.

[3] Keithley White Paper: Orthogonal Frequency Division Multiplexing, September 2008, vol. 14.

[4] 4G Americas White Paper: MIMO and Smart antennas for mobilebroadband systems, October 2012, vol. 138

[5] Zalitskiy A., Shel'gov V., Testirovaniye infrastrukturnykh resheniy dlya setey LTE: Zhurnal setevykh resheniy/Telekom, № 04, 2011.

[6] 4G v Ukraine: chto eto, kogda poyavitsya i na skol'ko podorozhayet svyaz' (INFOGRAFIKA). [Yelektronniy resurs]. - $\quad$ Rezhim dostupu: https://fakty.ictv.ua/ru/lifestyle/gadzhety/20180320-4g-vukrayini-shho-tse-koly-z-yavytsya-i-na-skilkyzdorozhchaye-zv-yazok/ 7. Geoff Huston, Best efforts networking, July 2001, vol 3 